\title{
Prospects of Fast-Neutron Resonance Radiography and its Requirements for Instrumentation
}

\author{
David Vartsky* \\ Soreq NRC \\ Yavne 81800, Israel \\ E-mail: davidasoreq.gov.il
}

Fast neutron resonance radiography (FNRR) is an imaging method that exploits the characteristic crosssection structure of different isotopes in the energy-range $E_{n}=1-10 \mathrm{MeV}$. FNRR holds promise for detecting a broad range of conventional and improvised explosives, by determining the identity and density distribution of light elements such as $\mathrm{C}, \mathrm{N}$ and $\mathrm{O}$ within an inspected object. FNRR is also employed to locate diamonds in a mineral rock environment.

This contribution reviews the various techniques of FNRR and examines their advantages and shortcomings. The required FNRR system characteristics for detection of explosives, such as detection efficiency, spatial resolution and energy resolution are outlined and some recent solutions aimed at achieving these goals are presented.

International Workshop on Fast Neutron Detectors

University of Cape Town, South Africa

April 3-6, 2006

\section{${ }^{*}$ Speaker}




\section{Introduction}

Fast Neutron Resonance Radiography (FNRR) is a fast-neutron transmission imaging method that exploits characteristic energy-variations of the total scattering cross-section in the $E_{n}=1-10 \mathrm{MeV}$ range to determine the identity and density distribution of specific elements (particularly, low Z) within an inspected object.

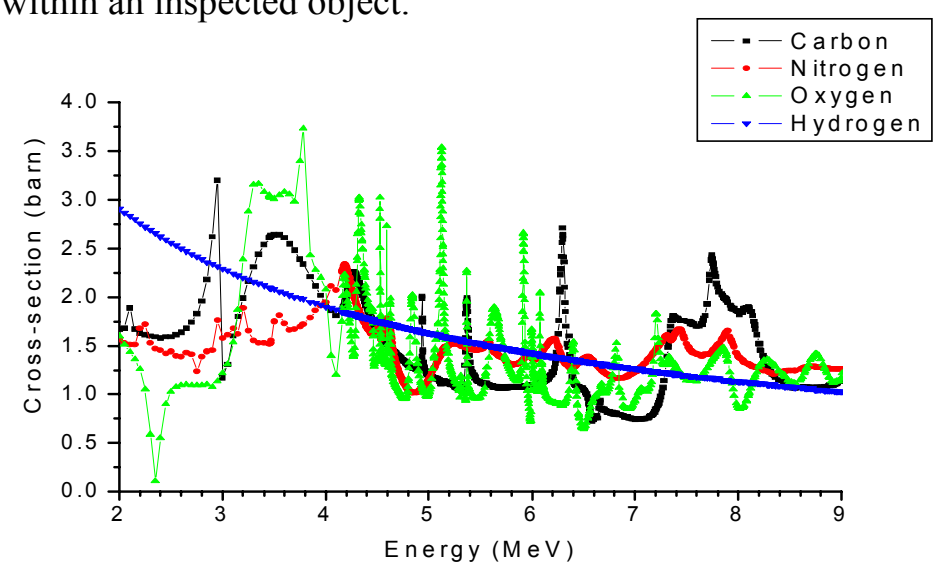

Fig. 1 Total neutron cross section of carbon, oxygen, nitrogen and hydrogen.

Fig. 1 shows the total cross-sections vs neutron energy for carbon, oxygen nitrogen and hydrogen. As can be observed there are sharp energy -fluctuations in values for $\mathrm{C}, \mathrm{O}$ and $\mathrm{N}$.

A principal application of FNRR is automatic detection of explosives,[1,2] in commercially transported items. Due to its ability to detect simultaneously the main elements present in explosives, such as $\mathrm{C}, \mathrm{O}$ and $\mathrm{N}$, the method is suitable for detection of most standard and improvised explosives. One of the improvised explosives employed recently by suicide bombers in various locations worldwide is Tri-Acetone-TriPeroxide (TATP). TATP is a high explosive which can be made from common household and readily procurable chemicals: acetone, hydrogen peroxide, and sulfuric acid. FNRR

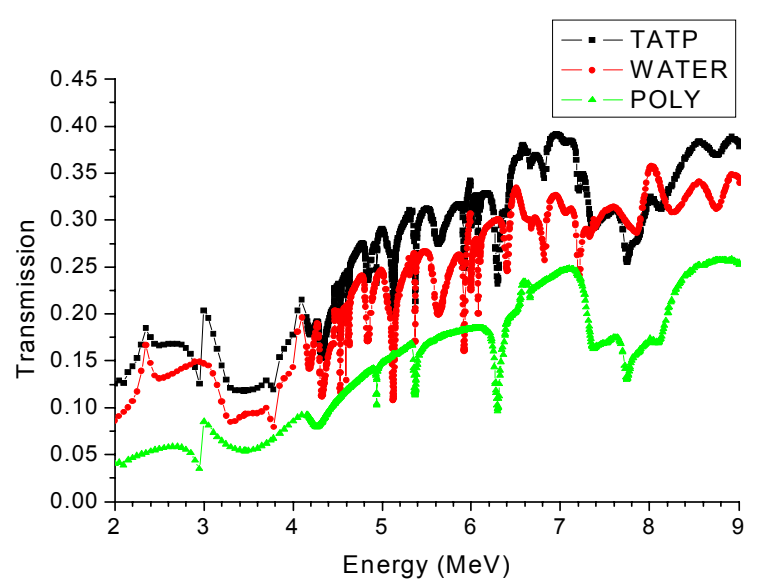

Fig. 2 Calculated transmission through 10 $\mathrm{cm}$ thick TATP, water and polyethylene can be particularly useful for detection of small quantities of TATP.

Fig. 2 shows a calculated transmission through $10 \mathrm{~cm}$ thick objects made of TATP, water and polyethylene. Due to different proportions of the constituent elements in each of these materials, the neutron transmission spectrum shows different features and can be used for distinguishing TATP from other materials of similar physical density. 
Another attractive application of FNRR is detection of diamonds in kimberlite rock[3]. Here the presence of carbon is detected in a rock which principally contains elements such as $\mathrm{Si}$, $\mathrm{O}, \mathrm{Al}$, Ti and $\mathrm{Fe}$.

In both the above applications, the precise knowledge of the neutron energy is a prerequisite for resonance radiography. There are 3 approaches to achieve this goal:

- Variable quasi-monoenergetic beam FNRR [4]. In this approach, continuous neutron energy variability is achieved by viewing the neutron source from different angles

- Switchable quasi-monoenergetic beam FNRR [3,5]. In this approach, toggling between two discrete neutron energies is performed.

- Pulsed broad energy beam FNRR (PFNTS) [6-9]. In this approach, a pulsed (ns) broad-energy neutron beam is used in combination with time-of-flight (TOF) neutron spectroscopy

The first two approaches can utilize un-pulsed (DC or CW) neutron beams, whereas the third approach requires a ns-pulsed neutron beam.

This contribution outlines the general requirements for FNRR and assesses how close each of the above variants of FNRR comes to meeting these requirements.

\section{FNRR instrumentation requirements}

\subsection{Neutron energy resolution}

The required energy resolution of the system is dictated by the characteristic width of the dips and peaks in the cross section structure (see Fig. 1). The system energy resolution influences the contrast with which a specific element is detected. With some exceptions, the required energy resolution is high at lower energies and broadens with neutron energy. For each element the requirements are somewhat different. Thus for oxygen the range is $\mathbf{1 0 - 3 0 0 ~} \mathbf{k e V}$, for nitrogen 50-100 keV and for carbon 50-500 keV.

\subsection{Neutron source dimensions}

The dimensions of the neutron source influence the quality of the radiographed image. Thus, in order to be able to reliably detect thin sheet explosives, the source dimensions should be of the order of 3-5 $\mathbf{~ m m}$.

\subsection{Required neutron flux}

It is important to estimate the fast neutron flux incident on the detector required for an FNRR-based explosives detection system (EDS). This parameter dictates the type of neutron source and its distance from the detector. The required neutron flux is dependent on many parameters, such as:

- Type, size and weight of the threat object

- Quantity and type of the surrounding cargo

- Detector efficiency

- Required inspection time

- Required detection probability and acceptable false alarm rate 
In order to estimate the required neutron flux a calculation was performed for the following scenario: detection of $200 \mathrm{~g}$ of TATP (density $=1 \mathrm{~g} / \mathrm{cc}, \mathrm{C} / \mathrm{O}=1.13$ ) hidden in an oversized package (80x80x50 $\mathrm{cm}^{3}$ in dimensions) filled with cotton textiles (density $0.3 \mathrm{~g} / \mathrm{cc}$, $\mathrm{C} / \mathrm{O}=1.29$ ). The inspection time is $10 \mathrm{~s}$ and the required detection probability and false alarm rate are $95 \%$ and $1 \%$ respectively. The calculation was based on using 4 energy bins centered on 2.7, 3.6, 6.8 and $7.8 \mathrm{MeV}$, which correspond to strong fluctuations in the cross-sections of oxygen and carbon. It was assumed that the incident flux in each energy bin is the same. The detection efficiency of a detector with dimensions $20 * 160 * 5 \mathrm{~cm}^{3}(\mathrm{w} * \mathrm{~h} * \mathrm{t})$ was taken as $37 \%$, $30 \%, 25 \%$ and $20 \%$ for the above energies, respectively. The calculations indicated that, in order to fulfill the above requirements, the unattenuated neutron flux incident on the detector should be about $1 * 10^{4} \mathbf{n} / \mathbf{s} / \mathbf{c m}^{2}$ for each energy bin. This calculation compares the $\mathrm{C} / \mathrm{O}$ ratio and is based on statistical considerations only. Clearly, other system-related factors which have not been taken into account here, such as various sources of background, number of views etc. will affect the result.

\subsection{Neutron detector requirements}

In most FNRR systems there is a need for a large area, efficient imaging detector. The dimensions and the detection efficiency will influence the inspection time of the object. The spatial resolution (pixel size) determines the minimal detectable threat size. Other important parameters are energy resolution (where applicable), counting rate capability and sensitivity to gamma-rays and scattered radiation. Table I summarizes the requirements of a FNRR detector.

Table I: Properties of a FNRR detector

\begin{tabular}{|l|l|}
\hline Parameter & Value \\
\hline \hline Detector area $\left[\mathrm{cm}^{2}\right]$ & $>50 \times 50$ \\
\hline Efficiency $[\%]($ at $8 \mathrm{MeV})$ & $>15$ \\
\hline Position resolution $[\mathrm{mm}]$ & $\sim 1$ (for sheet detection) \\
\hline Energy resolution $[\mathrm{keV}]$ & $10-500$ (for TOF detector) \\
\hline Count rate capability $\left[\mathrm{c} / \mathrm{s} / \mathrm{cm}^{2}\right]$ & $\sim 10^{5}$ \\
\hline Sensitivity to gamma-rays & As low as possible \\
\hline
\end{tabular}

\section{Review of FNRR techniques}

In this section we shall review the different approaches to FNRR and examine how well they fulfill the required conditions. The techniques are divided into two categories: un-pulsed and pulsed FNRR.

\subsection{Un-pulsed FNRR}

\subsubsection{Variable quasi-monoenergetic beam FNRR}

This method was developed by the MIT-LLNL collaboration[4,10]. In this approach the neutrons are produced by the $\mathrm{D}(\mathrm{d}, \mathrm{n})^{3} \mathrm{He}$ reaction with $2.3-2.5 \mathrm{MeV}$ deuterons on a $4 \mathrm{~atm}$. gas target. Due to the kinematics of the reaction the neutron energy varies continuously with the emission angle as shown in Fig. 3a. 

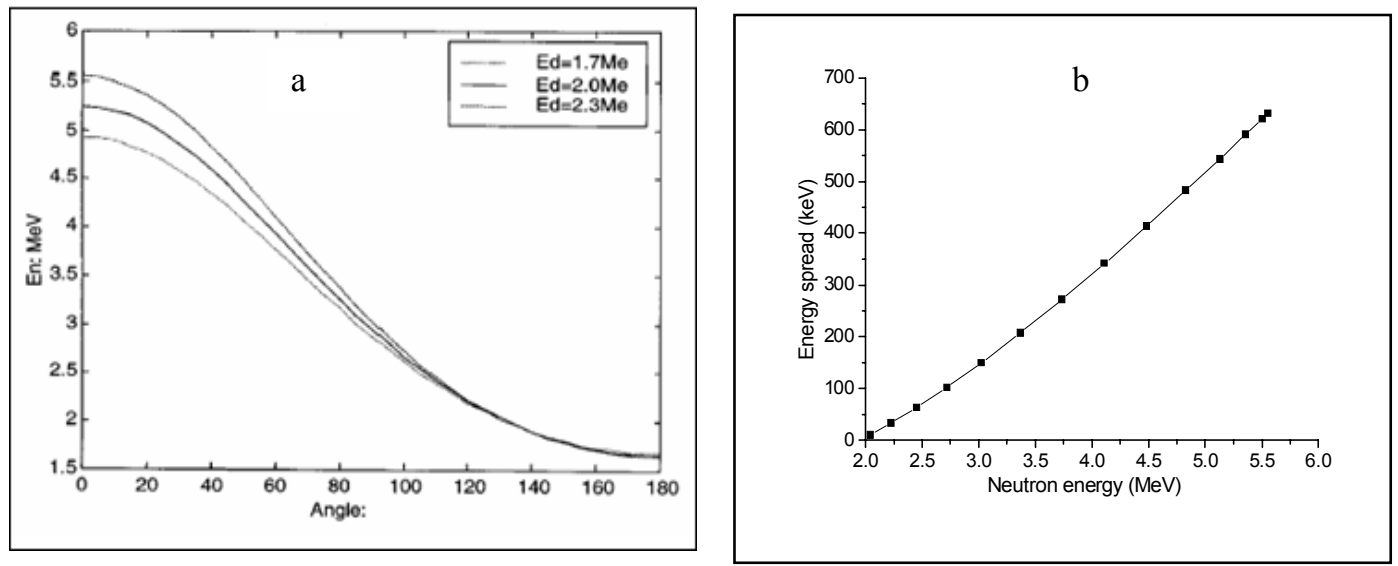

Fig. 3 a) Neutron energy variation vs emission angle [4] and b) Energy spread vs neutron energy

Thus the neutron energy is about $5.6 \mathrm{MeV}$ and $2.0 \mathrm{MeV}$ for emission angles of $0^{\circ}$ and $130^{\circ}$ respectively. For a small angular aperture at a given angle, the neutrons produced in this reaction can be considered mono-energetic, however due to the deuteron beam energy loss of about $600 \mathrm{keV}$ in the gas target, the neutron spectrum has a spread which is angle dependent. Fig. $3 \mathrm{~b}$ shows the energy spread vs neutron energy. It can be observed that the energy spread varies from about $10 \mathrm{keV}$ to about $630 \mathrm{keV}$ over the energy range $2-5.6 \mathrm{MeV}$.

The size of the gas target is about $\mathbf{1} \mathbf{~ c m}$ diameter by $\mathbf{1} \mathbf{~ c m}$ length[11] and the neutron flux at $1 \mathrm{~m}$ distance from the target is about $10^{4} \mathbf{n} / \mathbf{s} / \mathbf{c m}^{2}$. The magnitude of the flux decreases rapidly with the emission angle and at $90^{\circ}$ it drops down to about $10 \%$ of the $0^{\circ}$ value [12].

Resonant radiography is performed by positioning the inspected object and detector at $\mathbf{1 0}$ selected angles, that correspond to energies of specific resonances in $\mathrm{C}, \mathrm{O}$ and $\mathrm{N}$. Due to finite detector dimensions there is an energy variation across the detector and the angular aperture subtended by the detector must therefore be less than $10^{\circ}$. This restriction limits the dimensions of the inspected object.

The neutron detector consists of a plastic scintillator slab viewed by a liquid nitrogencooled low-noise CCD camera. Since the detector was incapable of discriminating between neutron events and gamma-rays emitted from the source, the elemental contrast was degraded and the experimentally-derived attenuation coefficients deviated substantially from published data. In order to reduce the gamma-ray yield a new LLNL source design was implemented using tungsten as the dominant source material [11].

Recently, a different detection approach was proposed[11], using 1-dimensional arrays of discrete liquid or solid scintillators operating in counting mode with pulse shape discrimination for separation of neutrons from gamma-rays. However the variation of neutron energy across the array appears to present a problem.

\subsubsection{Switchable quasi-monoenergetic beam FNRR}

This variant of the FNRR method was proposed by the De-Beers company, South Africa, for detection of diamonds in kimberlite rock[3,5]. Due to commercial reasons, not much information about this system is available. A significant amount of development work on the 
neutron source, detectors and system simulation has been performed in collaboration with the group of the University of Witwatersrand, SA[13-15].

In this application only the detection of carbon is of interest, therefore only two neutron energies, that correspond to the broad cross-section fluctuations around $6.8 \mathrm{MeV}$ and $7.8 \mathrm{MeV}$ are considered.

The neutron beam is produced by switching the deuteron energy between 4 and $5 \mathrm{MeV}$ using a $4 \mathrm{MeV}$ RFQ accelerator followed by a second accelerating stage, which can either transmit the deuteron beam, or boost its energy to $5 \mathrm{MeV}$. Neutrons are produced by interaction of the deuteron beam with deuterium gas target. Similarly to neutron beams produced in the previous system the spread of the neutron energy is about $600 \mathrm{keV}$ for the windowed gas target.[14]. This large energy spread is of no consequence, since the dip and peak in the carbon cross-section at 6.8 and $7.8 \mathrm{MeV}$ are both broad.

The detection system consists of a few centimeter thick scintillating fiber detector viewed either by a CCD camera or coupled to an amorphous Si plate[3].

\subsection{Pulsed broad beam FNRR (PFNTS)}

The Pulsed Fast Neutron Transmission Spectroscopy (PFNTS) method was proposed and first studied by the Oregon University group $[1,16,17]$, subsequently refined and taken through several blind tests for the FAA by Tensor-Technology, Inc [18-20], with some critical evaluation work performed by the Argonne NL group [21]. In the PFNTS method, a ns-pulsed broad energy $(1-10 \mathrm{MeV})$ neutron beam is incident on the inspected object and the transmitted neutron spectrum is measured by Time-of-Flight (TOF) method.

\subsubsection{Fast-Neutron Spectroscopy via Time-of-Flight}

To gain an understanding of the influence of detector properties on performance characteristics of an FNRR inspection system, it is instructive to consider the basics of the TOF method for measuring neutron energy. In TOF, an accelerator ion-beam is pulsed to generate a short (1-2 ns) neutron burst via a nuclear reaction. The time $\boldsymbol{t}_{\text {ToF }}$ required by individual neutrons in a pulse to reach a neutron detector positioned at a fixed distance from the source is related to neutron energy $\boldsymbol{E}_{\boldsymbol{n}}$ (within the non-relativistic energy range) by the simple relation:

$$
\boldsymbol{t}_{\text {TOF }}=d \sqrt{\frac{m}{2 E_{n}}}
$$

where $d$ is the source-detector distance, $m$ the neutron mass.

The overall time resolution is determined by a convolution of the beam burst duration and the instrumental time response of the detector.

\subsubsection{Single event TOF vs integrative TOF method}

Single-event-TOF (SETOF) is the conventional, most widely used TOF technique. In this method the arrival time of an individual neutron at the detector is recorded since its creation in the target during the beam burst. 
The time resolution is determined by the convolution of beam burst duration (1-1.5 ns), with uncertainties due to detector thickness $(\sim 0.25 \mathrm{~ns} / \mathrm{cm})$ and processing electronics $(<1 \mathrm{~ns})$. Thus, for a $1 \mathrm{~cm}$ thick detector, a typical overall time resolution is about $1.8 \mathrm{~ns}$. The energy resolution is dependent on the flight distance. For a $12 \mathrm{~m}$ long flight path, the above time resolution will translate to energy resolution of $12 \mathrm{keV}$ and $96 \mathrm{keV}$ for $2 \mathrm{MeV}$ and $8 \mathrm{MeV}$ neutrons respectively.

In the classical SETOF approach using Time-to Amplitude (TAC) or Time-to Digital (TDC) converters, which are characterized by dead times of several $\mu$ s following a registered event, it is essential that the probability for a neutron to be detected in a single accelerator beamburst is low $(<\sim 10 \%)$ to ensure that the probability for two events per burst is negligible. The reason is that only the first hit will be counted, resulting in spectrum distortion and counting losses. This restriction severely limits detector counting rates to tens of $\mathrm{kHz}$ and does not permit operation at high neutron flux intensities.

In recent years, with the advent of digital signal processing (DSP) it is possible to record the time of more than one event per burst. Such systems will be able to operate in the $\mathrm{MHz}$ counting rate range.

As opposed to SETOF mode, the integrative (ITOF) technique integrates the detector signal (light or current) during a well-defined gate time at a pre-selected $t_{\mathrm{TOF}}$ corresponding to a pre-selected energy bin, e.g., the energy-interval spanning a cross-section resonance. This is essentially stroboscopic photography of neutrons arriving at the detector on a nanosecond scale. The TOF spectrum is obtained by varying the delay of the integrating gate relative to the time of the burst.

The energy resolution is dictated by the beam burst duration, integrating gate width and time response of the detector. In case of a scintillation detector, the time response is determined by the light decay following an interaction with a neutron. For good time resolution, the time decay constant should be of the order of 1-2 ns. Clearly, only plastic or liquid organic scintillators can fulfill this requirement. The integrating gate width is instrumentally limited at present to about 2-5 ns. Thus the overall time resolution with this method is in the range of $5 \mathrm{~ns}$. This corresponds to an energy resolution of $33 \mathrm{keV}$ and $270 \mathrm{keV}$ for $2 \mathrm{MeV}$ and $8 \mathrm{MeV}$ neutrons respectively at a flight distance of $12 \mathrm{~m}$, significantly poorer than the resolution obtained with the SEC method.

On the positive side, the integrative data acquisition mode, will permit operation at essentially unlimited counting rates.

In summary the advantages of SETOF are: 1) Measurement of pure time of arrival distribution, without dependence on detector response and 2) The entire time spectrum is obtained simultaneously with very good time definition. The disadvantages are 1) Considerably reduced operating speed; 2) Increased data file size; 3) Usually coarse pixelization.

The advantages of ITOF are: 1) Permits operation at unlimited neutron fluxes; 2) Excellent spatial resolution. The disadvantages are: 1) Time resolution dictated by gate width 
and detector response; 2) Sequential scanning is required for accumulation of entire time spectrum

\subsubsection{Tensor Technology Inc. PFNTS system}

Tensor Technology Inc. has been developing the SETOF PFNTS method since 1992. In 1994, the FAA awarded the company a grant to build a multidimensional neutron radiometer (MDNR) aviation security system, which underwent a blind test in 1997 using a linear accelerator at the University of Kentucky. These studies indeed confirmed the considerable promise of the method for an EDS and in 1998 Tensor proposed an airport EDS system using an Ebco Technologies TR9D cyclotron as a pulsed neutron source. Nevertheless, the National Academy of Science panel in 1999[22], recommended not to deploy the PFNTS system at airports since poor system spatial resolution was not adequate for ensuring reliable detection of thin sheet explosives, the false alarm rate was high and size, weight and safety aspects were an issue. It is however noteworthy that the panel did explicitly stress that its conclusions were primarily dictated by the lack of supporting technologies (at the time of writing), rather than by any inherent shortcomings of the PFNTS method itself.

Since then Tensor has made significant progress in reducing the size of the accelerator, in the TOF data acquisition and also in detection algorithms.

The new neutron source proposed by Tensor is a T-T neutron generator being developed by the LBNL Plasma and Ion Source Technology group led by K.N. Leung[23]. The T-T reaction provides a flat neutron spectrum between $0-9 \mathrm{MeV}$. In this generator a point source is created by generating the plasma in a toroidal-shaped plasma chamber with 20 beamlets being extracted toward the central axis of the chamber, where a small diameter $(\sim 5 \mathrm{~mm})$, water-cooled hollow target tube is maintained at $120 \mathrm{kV}$ potential. The beam will be pulsed with $\mathbf{5 n s}$ bursts and $\mathbf{0 . 2 5 \%}$ duty factor. With such duty factor the expected flux at $1 \mathrm{~m}$ is about $\mathbf{2 \times 1 0 ^ { 4 }} \mathbf{n} / \mathbf{s} \mathbf{~ c m}^{2}$. This pulse duration appears to be too long for a high quality TOF measurement and will dominate the time resolution. The neutron flux appears to be inadequate for an operational system which will employ TOF flight path of 3-6 m. The expected generator dimensions are $100 \mathrm{~cm}$ long and $50 \mathrm{~cm}$ in diameter.

Tensor uses a large area detector [24] (see Fig. 4) consisting of individual $4 \times 4 \times 3$ $\mathrm{cm}^{3}$ plastic scintillators coupled to a photomultiplier tube. With such detector dimensions, the pixel size is $\mathbf{3 . 3 \times 3 . 3} \mathbf{c m}^{2}$ at the object position. Tensor has also made significant advances in time of flight data acquisition techniques and claims that each detector can now operate at an average rate of up to $\mathbf{5} \mathbf{~ M H z}$ with zero dead time [25]. This is certainly a breakthrough in SETOF

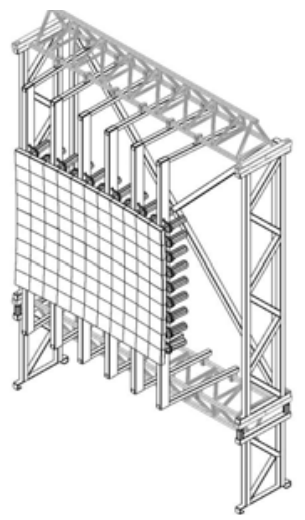

Fig. 4 Drawing of Tensor's detector array [24] data acquisition. 


\subsubsection{Improved resolution PFNTS detection systems}

It would appear that Tensor's detector spatial resolution is still inadequate for detection of thin sheet explosives. The two detection systems described in this section attempt to solve this problem by virtue of their capability to guarantee a sub-mm position resolution.

\subsubsection{High spatial resolution SETOF detector}

This recent idea was proposed by Dangendorf et al. and is described in detail elsewhere in these proceedings[26]. Rather than viewing the individual scintillators with a phototube, each detector is viewed by a pulse counting image intensifier.

Fig. 5 schematically illustrates the principle of operation. The individual scintillator fiber screen is optically coupled to a pulse counting image intensifier which measures the TOF and position of each detected neutron.

The time and position resolution achievable in this method is $<\mathbf{1} \mathbf{~ n s}$ and about $100 \mu \mathrm{m}$, respectively. In the above work, the data acquisition has been taken in a list mode, the relevant parameters such as position and TOF of each individual event were measured and stored in a multi-dimensional histogram. The advantage of this method is that very good parameter definition can be achieved, usually in subsequent off-line analysis. This is, however, at the expense of considerably reduced operating speed as well as increased data file size. For a practical device a dedicated high speed acquisition system will have to be

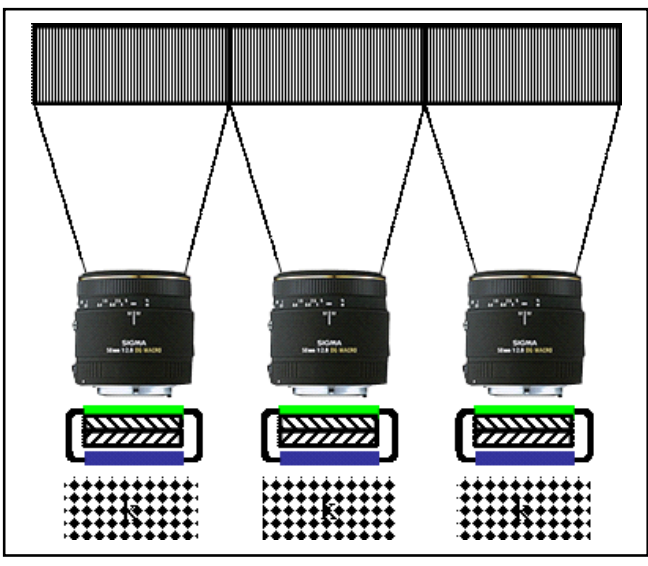

Fig. 5 Schematical description of high spatial resolution SETOF detector used for a on-line analysis.

\subsubsection{High spatial resolution ITOF detector}

The time-resolved integrative optical neutron (TRION) detector is developed by the Soreq /PTB collaboration $[8,9,28]$. The concept of TRION, first proposed by Dangendorf et al in 2002 [27] is shown in Fig. 6. The pulsed neutron beam impinges on a $30 \mathrm{~mm}$ thick scintillating fiber screen, causing the emission of light. The light is reflected by a front-coated bending mirror (98\% reflectivity, positioned at an angle of $45^{\circ}$ relative to the neutron beam direction) towards a large aperture $120 \mathrm{~mm}$ F\#0.95 lens. The light is amplified by a ns-gated image intensifier and transmitted by a relay lens to a cooled CCD camera.

The gated image intensifier acts as a fast electronic shutter for the CCD camera. The gate signal is created by a computer controlled gate $\&$ delay generator (GDG) at a delay relative to the accelerator beam burst. In a typical pulsed fast-neutron beam, the repetition rate is of the order of $2 \mathrm{MHz}$. Within a time interval of $500 \mathrm{~ns}$, depending on 
neutron-source to detector distance, the system should integrate neutrons into an image in a well defined time window. Typical gate width is 2-10 ns. A cooled CCD camera, captures the image from the image intensifier through a relay lense. The camera integrates the image over many cyclotron pulses, until sufficient counting statistics in the pixels of the CCD is obtained. The TOF spectrum is accumulated by varying the gate delay.

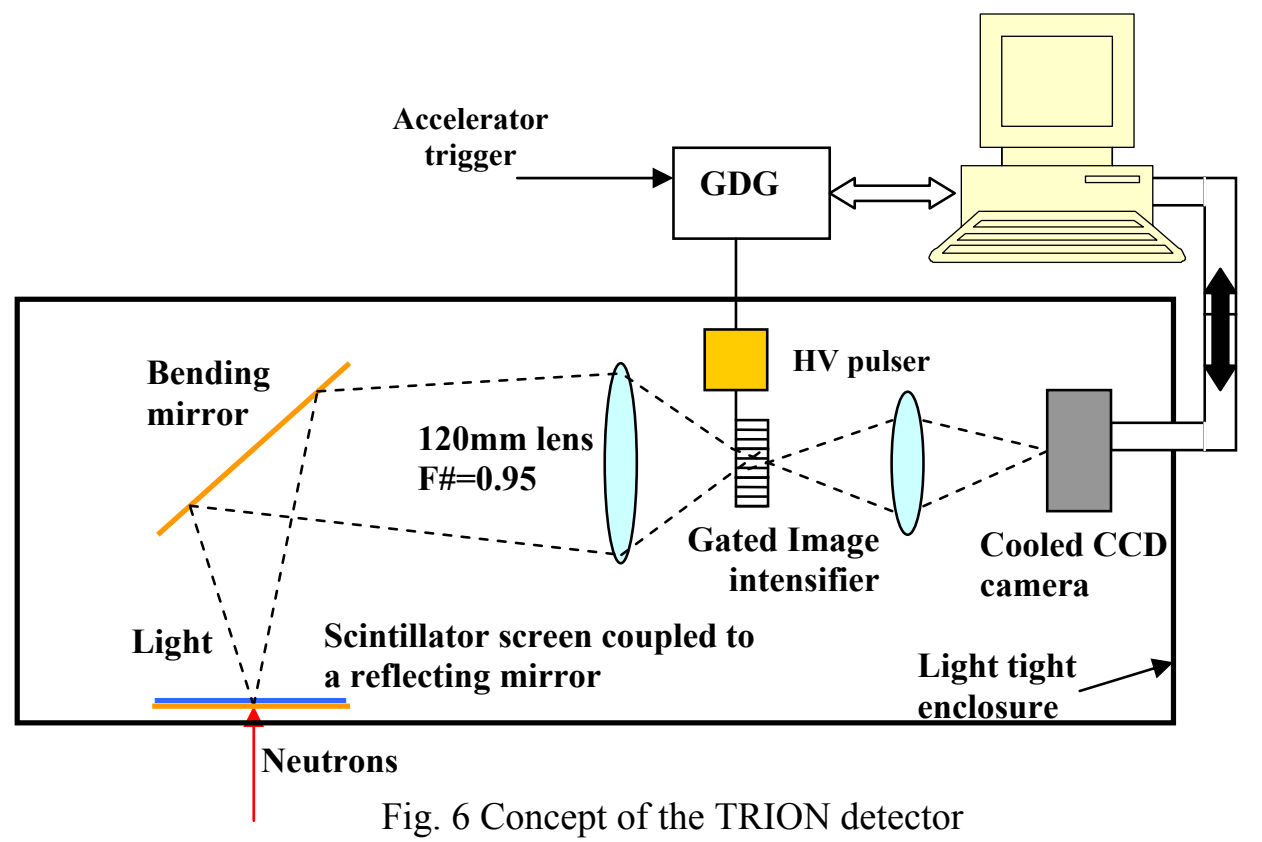

The position resolution of TRION is dictated mainly by the fiber dimensions and is about $0.5 \mathrm{~mm}$. Fig. 7a shows an image obtained by taking a fast neutron radiograph of objects (Fig.7b) concealed behind a 1" thick lead shield (Fig. 7c). The entire neutron spectrum was integrated to create this image. Clearly, the position resolution is very good and will permit visual examination of the inspected object contents in addition to the automatic element specific explosives detection. The high spatial resolution will aid in detecting thin shape explosives.
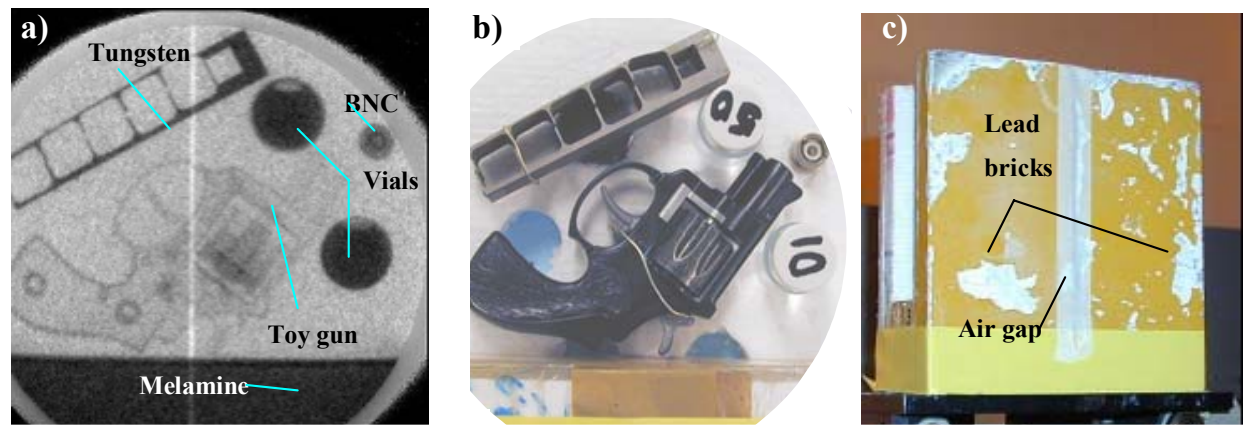

Fig. 7. Fast neutron radiograph (a)of objects shown in (b) behind a 1" thick $\mathrm{Pb}$ shield

The time of flight resolution of the TRION detector is dependent on the gating pulse width and the light decay time constant of the scintillating screen. In the present system the minimal 
gate width that permitted a reliable full opening of the image intensifier was $8 \mathrm{~ns}$. This width is limited by photocathode impedance and the ns-rise-time HV pulse transmission system to the image intensifier and in principle can be reduced further. It is expected that time resolutions of 2-5 ns are realistic for TRION.

Fig. 8 shows a TOF spectrum of neutrons transmitted through $10 \mathrm{~cm}$ thick graphite block taken with TRION (black line) and with a plastic scintillator NE102 operated in event counting mode (red line). As can be observed some of the contrast obtained in ITOF mode has been lost, especially for narrow resonances. Methods of recovery of this contrast are being investigated at present.

Fig. 9 shows images of objects made of carbon, melamine and steel taken in 6 different TOF-bins. Top pictures show radiographs of a phantom (bottom left) made of a melamine block, carbon rods and steel wrench. Lower right pictures show reconstructed pure images of $\mathrm{C}$ and $\mathrm{N}$.

Currently, TOF imaging by TRION is only capable of capturing a single TOF-bin image per neutron burst.. Thus, in order to progress towards a real-time operational system, it is necessary to develop a detector capable of capturing several different TOFbin images for each neutron burst. This can be achieved by employing several nstriggered CCD cameras viewing an un-gated

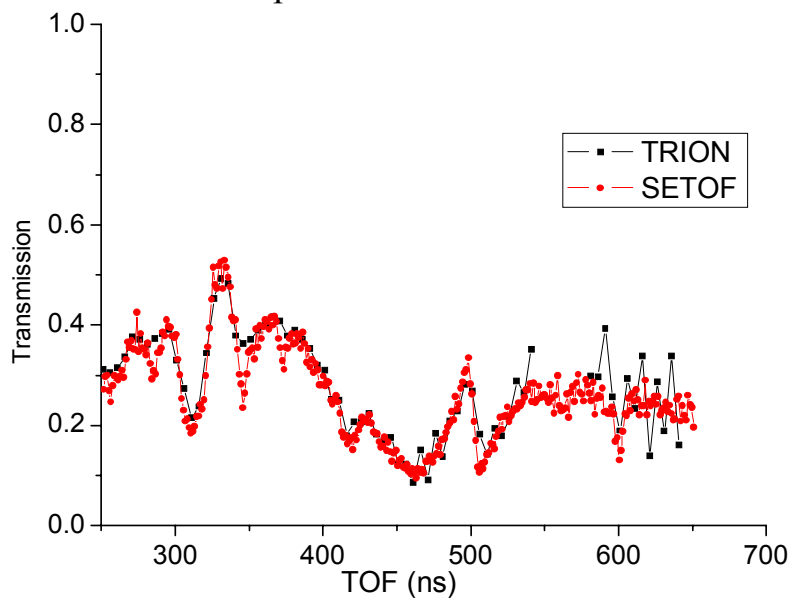

Fig. 8 Transmission TOF spectra for a $10 \mathrm{~cm}$ thick block of graphite obtained in ITOF (black) and SETOF (red) mode

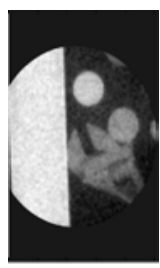

$180 \mathrm{~ns}$

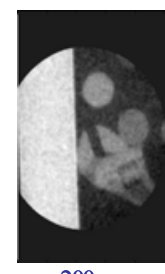

$200 \mathrm{~ns}$

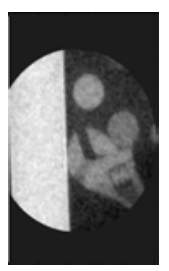

$210 \mathrm{~ns}$

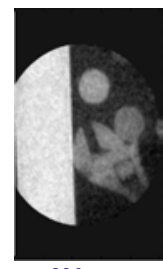

$230 \mathrm{~ns}$

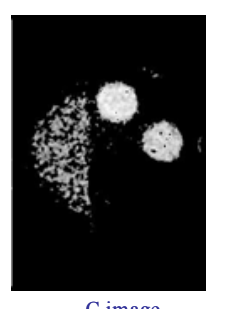

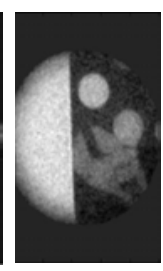

$320 \mathrm{~ns}$

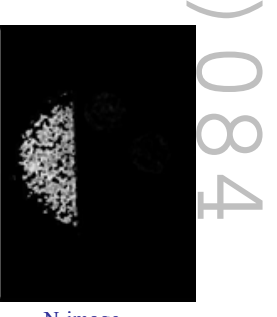

Nimage

Fig. 9 Neutron radiographs of melamine, carbon and steel objects in 6 TOF-bins

fast image intensifier, such that each camera acquires a different time/energy interval per accelerator pulse. The number of cameras will be determined according to the minimal number of cross-section peaks and dips required for multi-elemental imaging[29]. This is the subject of our present work.

\section{Summary and conclusions}

In this section we present a summary of the characteristics of the discussed FNRR variants. Only multi-energy explosives detection methods are compared. Since the diamond detection method concentrates on measurement of two energy bins only, it is not discussed here. Table II shows the performance of each FNRR variant. The color code is: green-good, red-bad, orange-medium. 
Table II Comparison of FNRR methods for detection of explosives

\begin{tabular}{|c|c|c|c|c|}
\hline \multirow[b]{2}{*}{ Parameter } & \multirow{2}{*}{$\begin{array}{c}\text { Variable } \\
\text { energy } \\
\text { beam }[10]\end{array}$} & \multicolumn{3}{|c|}{ Pulsed broad energy beam } \\
\hline & & SETOF[20] & $\begin{array}{l}\text { SETOF high } \\
\text { resolution[26] }\end{array}$ & ITOF $[8,9]$ \\
\hline Energy range $[\mathrm{MeV}]$ & $1-6$ & $1-8$ & $1-10$ & $1-10$ \\
\hline Penetration & Limited & Sufficient & Sufficient & Sufficient \\
\hline Energy resolution & Medium & Excellent & Excellent & Medium \\
\hline No. of energy bins & Limited & Un-restricted & Un-restricted & Limited \\
\hline Detection efficiency & Adequate & Adequate & Adequate & Adequate \\
\hline Position resolution [mm] & $5-10$ & $40-50$ & 0.5 & 0.5 \\
\hline Sensitivity to $\gamma$-rays & yes & no & no & no \\
\hline Scattered neutron rejection & no & yes & yes & yes \\
\hline Count rate capability & Unlimited & Limited & Limited & Unlimited \\
\hline TOF path (several meters) & No & Yes & Yes & Yes \\
\hline Pulsing required & No & Yes & Yes & Yes \\
\hline Complexity & No & Yes & Yes & Yes \\
\hline
\end{tabular}

FNRR is suitable for inspection of medium-size objects $(100 \times 100 \times 100 \mathrm{~cm} 3)$. It may be used for inspection of aviation containers loaded with bags and non-dense cargo, but its penetration for dense hydrogenous cargo will be limited. With its inherent multi-elemental detection capability and the recently improved spatial resolution, it has a very high potential for detection of standard and improvised explosives (including thin sheet).

\section{References}

[1] J.C. Overley, Determination of $H, C, N, O$ content of bulk materials from neutronattenuation measurements Int. J. Appl. Rad. \& Isot. 36 (1985)

[2] T.G. Miller and R.A. Krauss Substance identification using neutron transmission, SPIE Proceedings, Vol. 2511 (1995) 14,

[3] U.A.S. Tapper and G.W. Over, Neutron detection apparatus, World Patent WO0029873, 25 May 2000.

[4] G. Chen and R. C. Lanza, Fast neutron resonance radiography for elemental imaging: theory and applications, IEEE Trans. Nucl. Science 49 (2002) 1919

[5] R.W.Hamm, C.W. Franklyn, J. Guzek, B.R. Kala, U.A.S. Tapper and J.I.W. Watterson, Characterization of a variable energy deuteron $R F Q$ system for neutron production, http://www.anl.gov/conferences/LINAC98/papers/TH4106.pdf

[6] J.C. Overley, Explosives detection through fast-neutron time-of-flight attenuation measurements, Nucl. Instr. and Meth. B 99 (1995) 728

[7] T.G. Miller, P.K. Van Staagen, B.C. Gibson, J.L. Orthel and R.A. Krauss, Contraband detection using neutron transmission, SPIE Proceedings, Vol 2936 (1997) 102.

[8] V. Dangendorf, C. Kersten, G. Laczko, D. Vartsky, I. Mor, M.B. Goldberg, G. Feldman, O. Jaguztky, U. Spillman, A. Breskin, R. Chechik, Detectors for energy resolved fast neutron imaging, Nucl. Instr. and Meth. A 535(2004) 93

[9] D. Vartsky, I. Mor, M.B. Goldberg, I. Mardor, G. Feldman, D. Bar, A. Shor, V. Dangendorf, G. Laczko, A. Breskin and R. Chechik, Time Resolved Fast Neutron Imaging: Simulation of Detector Performance Nucl. Instr. Meth. A542 (2005) 197 
[10]W.L. Raas, B.W. Blackburn, E. Boyd, J. Hall, G. Kohse, R.C. Lanza, B. Rusnak and J.I.W. Watterson, Neutron resonance radiography for explosives detection: technical challenges, IEEE Trans. Nucl. Science, in print (2005) 129.

[11]R.C. Lanza, Fast neutron resonance radiography for threat interdiction: technical challenges and preliminary results, these proceedings

[12]J.B. Marion and J.L. Fowler Fast Neutron Physics, Part I, Interscience Publishers Inc., New York, (1960) 80

[13]I.J.Watterson and R.M. Ambrosi, Some fundamental considerations in resonance imaging using fast neutrons, Nucl. Instr. and Meth. A 513 (2003). 367

[14]R.M. Ambrosi and Watterson, The effect of imaging geometry and the impact of neutron scatter on the detection of small features in accelerator-based fast neutron radiography, Nucl. Instr. and Meth. A 524 (2004). 340

[15]J. Guzek, K. Richardson, C.B. Franklyn, A. Waites, W.R. McMurray, J.I.W. Watterson and U.A.S. Tapper, Development of high pressure deuterium gas targets for the generation of intense mono-energetic fast neutron beams, Nucl. Instr. and Meth. B152 (1999). 515

[16]J.C. Overley, Element-Sensitive Computed Tomography with Fast Neutrons, Nucl. Instr. and Meth. B 24/25 (1987) 1058

[17]J.C. Overley, Explosives detection through Fast-Neutron Time-of-Flight Attenuation Measurements, Nucl. Instr. and Meth. B 99 (1995) 728

[18]T.G. Miller, Use of neutron tomography for airline security, SPIE Proceedings, Vol. $\underline{\mathbf{2 0 9 3}}$ (1994) 204.

[19]T.G. Miller, Drug and tobacco detection using neutron transmission/attenuation, SPIE Proceedings, Vol. 2276 (1994) 200.

[20]T.G. Miller, P.K. Van Staagen B.C. Gibson, J.L. Orthel and R.A. Krauss, Contraband detection using neutron transmission, SPIE Proceedings, Vol. 2936 (1997) 102.

[21]C.L. Fink , B.J. Miklich, T.J. Yule, P. Humm, L. Sagalovsky and M.M. Martin,Evaluation of neutron techniques for illicit substance detection, Nucl. Instr. \& Meth. B99 (1995) 748.

[22] The Practicality of PFNTS for Aviation Security", NAS Panel report (1999), http://books.nap.edu/html/aviation_spectroscopy/

[23]J. Reijonen, Compact neutron generators for medical, homeland security and planetary exploration, Proceedings of Paricle Accelerator Conference, 16-20 May, 2005, 49

[24]P.K. Van Staagen, T.G. Miller, B.C. Gibson and R.A. Krauss, High speed data acquisition for contraband identification using neutron transmission Proceedings of $14^{\text {nd }}$ International conference on application of accelerators in research and industry, Nov. 6-9 1996, Detection Technology Symp. and Aviation Security Technology Conf., November 12-15, 1996. http://www.tensor-technology.com/

[25] T.G. Miller and P.K. Staagen, (2004), private communication

[26]V. Dangendorf, F. Kaufmann, O. Jagutzki, J. Barnstedt, D. Vartsky and A. Breskin, Time resolved fast-neutron imaging with pulse-counting image intensifier, these proceedings.

[27] V. Dangendorf, C. Kersten, G. Laczko, O. Jaguztky, U. Spillman, Fast Neutron Resonance Radiography in a Pulsed Neutron Beam, $7^{\text {th }}$ World Conf. On Neutron Radiography, Rome, Sept. 2002, http://arxiv.org/abs/nucl-ex/0301001

[28]I. Mor, D. Vartsky, I. Mardor, M.B. Goldberg, D. Bar, G. Feldman and V. Dangendorf, Monte-Carlo simulations of time-resolved, optical readout detector for fast-neutron transmission spectroscopy, these proceedings.

[29] Submitted as a US patent, Filing date: 1 Nov. 2004 